(1)

CrossMark

\title{
Towards a close computed tomography monitoring approach for screen detected subsolid pulmonary nodules?
}

\begin{abstract}
Ernst T. Scholten ${ }^{1,2}$, Pim A. de Jong ${ }^{1}$, Bartjan de Hoop ${ }^{1}$, Rob van Klaveren ${ }^{3}$, Saskia van Amelsvoort-van de Vorst ${ }^{1}$, Matthijs Oudkerk ${ }^{4}$, Rozemarijn Vliegenthart ${ }^{5}$, Harry J. de Koning ${ }^{6}$, Carlijn M. van der Aalst ${ }^{6,7}$, René M. Vernhout ${ }^{6}$, Harry J.M. Groen ${ }^{8}$, Jan-Willem J. Lammers ${ }^{9}$, Bram van Ginneken ${ }^{10}$, Colin Jacobs ${ }^{10,11}$, Willem P.T.M. Mali ${ }^{1}$, Nanda Horeweg ${ }^{6,7}$, Carla Weenink $^{12}$, Erik Thunnissen ${ }^{13}$, Mathias Prokop ${ }^{14}$ and Hester A. Gietema ${ }^{1}$

Affiliations: 'Dept of Radiology, University Medical Center, Utrecht, The Netherlands. ${ }^{2}$ Dept of Radiology, Kennemer Gasthuis, Haarlem, The Netherlands. ${ }^{3}$ Dept of Pulmonology, Lievensberg Hospital, Bergen op Zoom, The Netherlands. ${ }^{4}$ Center for Medical Imaging - North East Netherlands, University of Groningen, University Medical Center Groningen, Groningen, The Netherlands. ${ }^{5}$ Dept of Radiology, University of Groningen, University Medical Center Groningen, Groningen, The Netherlands. ${ }^{6}$ Dept of Public Health, Erasmus Medical Center, Rotterdam, The Netherlands. ${ }^{7}$ Dept of Pulmonology, Erasmus Medical Center, Rotterdam, The Netherlands. ${ }^{8}$ Dept of Pulmonology, University of Groningen, University Medical Center Groningen, Groningen, The Netherlands. ${ }^{9}$ Dept of Pulmonology, University Medical Center, Utrecht, The Netherlands. ${ }^{10}$ Diagnostic Image Analysis Group, Radboud University Nijmegen Medical Center, Nijmegen, The Netherlands. ${ }^{11}$ Fraunhofer MEVIS, Bremen, Germany. ${ }^{12}$ Dept of Pulmonology, Kennemer Gasthuis, Haarlem, The Netherlands. ${ }^{13}$ Dept of Pathology, VU University Medical Center, Amsterdam, The Netherlands.

${ }^{14}$ Dept of Radiology, Radboud University Nijmegen Medical Center, Nijmegen, The Netherlands.
\end{abstract}

Correspondence: Pim A. de Jong, Heidelberglaan 100, 3584 CX Utrecht, The Netherlands.

E-mail: P.deJong-8Qumcutrecht.nl

ABSTRACT Pulmonary subsolid nodules (SSNs) have a high likelihood of malignancy, but are often indolent. A conservative treatment approach may therefore be suitable. The aim of the current study was to evaluate whether close follow-up of SSNs with computed tomography may be a safe approach.

The study population consisted of participants of the Dutch-Belgian lung cancer screening trial (Nederlands Leuvens Longkanker Screenings Onderzoek; NELSON). All SSNs detected during the trial were included in this analysis. Retrospectively, all persistent SSNs and SSNs that were resected after first detection were segmented using dedicated software, and maximum diameter, volume and mass were measured. Mass doubling time (MDT) was calculated.

In total 7135 volunteers were included in the current analysis. 264 (3.3\%) SSNs in 234 participants were detected during the trial. 147 (63\%) of these SSNs in 126 participants disappeared at follow-up, leaving 117 persistent or directly resected SSNs in 108 (1.5\%) participants available for analysis. The median follow-up time was 95 months (range 20-110 months). 33 (28\%) SSNs were resected and 28 of those were (pre-) invasive. None of the non-resected SSNs progressed into a clinically relevant malignancy.

Persistent SSNs rarely developed into clinically manifest malignancies unexpectedly. Close follow-up with computed tomography may be a safe option to monitor changes.

@ERSpublications

Persistent subsolid pulmonary nodules may be safely monitored with follow-up computed tomography http://ow.ly/CqWN1

The online version of this article has been corrected. The correction is outlined in the erratum published in the May 2015 issue of the European Respiratory Journal [DOI: 10.1183/09031936.50005914].

For editorial comments see Eur Respir J 2015; 45: 592-595 [DOI: 10.1183/09031936.00222914].

This article has supplementary material available from erj.ersjournals.com

Received: Jan 082014 | Accepted after revision: Sept 182014 | First published online: Nov 272014

Copyright @ERS 2015. 


\section{Introduction}

Lung cancer screening with computed tomography (CT) has increased the awareness of a specific subtype of pulmonary nodules: the subsolid nodule (SSN). Currently, there are limited data that provide evidence and guidance on how to manage these specific nodules $[1,2]$. An SSN is defined as a circumscribed area of increased lung attenuation with preservation of the bronchial and vascular margins and also referred to as a ground-glass nodule (GGN). An SSN can be a part-solid GGN (part of the nodule completely obscures the underlying lung parenchyma) or a pure GGN (no completely obscured areas, hence pure ground glass) [3]. While transient SSNs can represent a large range of benign diseases, persistent SSNs have a high likelihood of malignancy, with reported malignancy rates ranging from $19.4 \%$ to $75 \%[4,5]$. These malignancy rates are much higher than the likelihood of malignancy of solid pulmonary nodules: the Early Lung Cancer Action Project (ELCAP) study reported a malignancy rate among their positive findings of $34 \%$ for pure GGNs and $63 \%$ for part-solid GGNs, while only $7 \%$ of the solid nodules were malignant [1].

Despite their high malignancy rates, SSNs usually grow slowly with a low propensity of distant spread and have an excellent prognosis $[6,7]$. Data suggest that resected SSNs often represent adenocarcinoma in situ (AIS) [8-12] and less aggressive treatment may be suitable for these nodules, especially in patients with severe comorbidity.

For solid pulmonary nodules, a volume doubling time (VDT) $\geqslant 400$ days is often considered to reflect benign histology [13-17]. Diagnosis and treatment of these slow growing solid nodules is usually considered over-diagnosis: diagnosis of "disease" that will never cause symptoms or death during a patient's lifetime. Since SSNs usually have a slow growth rate with VDTs $\geqslant 400$ days, but with a high likelihood of malignancy, follow-up guidelines for solid pulmonary nodules are not appropriate for SSNs. In 2013, the Fleischner Society published recommendations for management of SSNs, but the committee emphasised that the recommendations were largely based on expert opinion [3]. To confirm persistence of SSNs, a three-month follow-up CT was advised. Persistent pure GGNs $>5 \mathrm{~mm}$ are recommended to be followed with an annual CT over at least three years. Persistent part-solid GGNs are recommended to be managed more aggressively, regardless of their size. For these nodules, further evaluation and resection was advised for patients without severe comorbidities.

In the Dutch-Belgian lung cancer screening trial (Nederlands Leuvens Longkanker Screenings Onderzoek (NELSON); International Standard Randomised Controlled Trial Number 63545820) an approach of close follow-up of persistent SSNs was chosen [18]. Only SSNs with a solid component $>500 \mathrm{~mm}^{3}$ at baseline were referred to a pulmonologist immediately. All other SSNs were followed. Further evaluation was only indicated in case of increase in size (either total size or size of the solid component) or density. It was our objective to evaluate our close follow-up protocol for SSNs and relate the results to the new recommendations [3].

\section{Methods}

\section{Study participants}

This is an ancillary study of NELSON trial, which was approved by the Dutch and Belgian Ministries of Health and by the ethical review boards of the participating hospitals [19]. Written informed consent was obtained from each participant. Details of the study population were described previously [19]. For the present study, we included all participants from the CT screening arm from the participating Dutch screening centres (University Medical Center Groningen, University Medical Center Utrecht and Kennemer Gasthuis, Haarlem, the Netherlands).

\section{CT scanning and reading protocol}

CT screening was done at baseline, 1 year and 3.5 years and 5.5 years after baseline plus additional follow-up CT examinations in case indeterminate nodules were detected [18]. All CTs were read for noncalcified nodules and detected nodules were characterised as solid nodule or SSN, either pure GGN or part-solid GGN. Participants who were referred to a pulmonologist underwent diagnostic work-up including a standard-dose CT with intravenous contrast, bronchoscopy and/or biopsy. Positron emission

Clinical trial: This study is registered at www.controlled-trials.com with identifies number ISRCTN63545820

Support statement: The NELSON trial is supported by Zorg Onderzoek Nederland-Medische Wetenschappen (ZonMw), KWF Kankerbestrijding, Stichting Centraal Fonds Reserves van Voormalig Vrijwillige Ziekenfondsverzekeringen (RvvZ), G. Ph. Verhagen Foundation, Rotterdam Oncologic Thoracic Study Group (ROTS) and Erasmus Trust Fund, Stichting tegen Kanker, Vlaamse Liga tegen Kanker and LOGO Leuven and Hageland.

Conflict of interest: Disclosures can be found alongside the online version of this article at erj.ersjournals.com 
tomography (PET) was not routinely included in the diagnostic work-up. Based on the results of these exams, the pulmonologist eventually decided with the participant whether resection of the SSN was needed.

\section{Evaluation of SSNs}

All participants who had a SSN $\geqslant 5 \mathrm{~mm}$ according to trial database on any screening CT were identified. Previous and later exams from these participants were reviewed by two (chest) radiologists with 30 and 10 years of experience, to detect possible unreported SSNs on previous or later CTs and to confirm the subsolid nature of the nodule.

\section{Size measurements}

During the trial, volumetry software (LungCare; Siemens Healthcare, Erlangen, Germany) was used to measure nodule dimensions [18]. This software was developed for solid nodules and often failed when applied to SSNs. Therefore, the diameter of SSNs was measured in the trial using electronic callipers. Based on results of these 2-dimensional measurements, volume was calculated by using the following equation:

$$
\text { Volume }=4 / 3 \pi \times(\text { diameter } / 2)^{3}
$$

When a solid component was present, the size of the solid component was quantified using the volumetry software. Increase in total volume or volume of the solid component $>25 \%$ was considered as significant growth in the original NELSON protocol. The radiologist was also allowed to refer a subject to the pulmonologist in case of visual impression of growth not supported by the measurements.

For the current analysis, we retrospectively measured volume and mass of all nodules with dedicated software, based on an established method for solid lesion segmentation, adapted for segmentation and volumetry of SSNs (CIRRUS Lung; Diagnostic Image Analysis Group, Nijmegen, the Netherlands, and Fraunhofer MEVIS, Bremen, Germany) [20]. For the present study, an increase of at least $30 \%$ of the mass of the nodule was considered as growth. This cut-off of $30 \%$ is based on our phantom study [21] and preliminary unpublished data on the interscan variation in our population.

Follow-up

Patient outcome was retrieved from the NELSON database that contained histological diagnosis, follow up and vital status until November 2012. If needed, hospital information systems were investigated for additional details.

\section{Statistics}

Growth was calculated by comparing actual mass to the mass at first detection and expressed as a percentage. Growth rate was expressed as mass doubling time (MDT) in days, using the following equation:

$$
\mathrm{MDT}=\Delta \mathrm{t} \times(\ln (2)) /(\ln (\mathrm{M} 2 / \mathrm{M} 1))
$$

where $\Delta \mathrm{t}$ is the time difference between the two measurements and M1 and M2 is the mass at first detection and sample measurement, respectively.

Data are presented as median and interquartile range (IQR) for non-parametric variables and as mean and standard deviation for normally distributed variables. A t-test was used to compare the subjects with an SSN to the total study population. A p-value $<0.05$ was considered significant.

Statistical analysis was performed with software (IBM SPSS version 20; SPSS, Chicago, IL, USA).

\section{Results}

\section{Study population and nodule characteristics}

In total 7135 volunteers (1152 females (16.1\%)) received at least a baseline CT. Median age at baseline was 58.0 years (IQR 8.0 years) and median smoking history was 38.0 pack-years (IQR 19.8 pack-years); 3194 participants $(44.8 \%)$ were still smokers at the date of inclusion.

264 SSNs in 234 (3.3\%) participants were detected during the trial. 147 (63\%) SSNs in 126 participants disappeared at follow-up, leaving 117 persistent SSNs in 108 (1.5\%) participants available for analysis, including 11 SSNs in eight participants who were referred after baseline CT and had their SSN resected. In five other participants with five nodules, changes in size could not be assessed because of missing data in three or inadequate segmentation of the nodule in two cases, leaving 101 nodules in 98 participants available for growth-rate analysis. Five participants had two SSNs, two participants had three SSNs. 81 persistent SSNs were visible at baseline and 36 became first visible at follow-up. Baseline demographics are shown in table 1. Median follow-up time since the first appearance of the nodule was 95 months (range 20-110 months). Median follow-up period after resection was 20 months (range 2-39 months). 
TABLE 1 Baseline demographics

$\begin{array}{lc}\text { Age at baseline years } & 62.0(10.1) \\ \text { Sex female } \mathbf{n}(\%) & 28(26) \\ \text { Smoking status } \mathbf{n}(\%) & 57(52.8) \\ \quad \text { Current } & 51(47.2) \\ \text { Former } & 38(19.8) \\ \text { Smoking history pack-years } & 69 \\ \text { Pure GGN at detection } & 11.1 \pm 3.6(5.1-22.6) \\ \quad \text { Number } & \\ \quad \text { Size mm } & 48 \\ \text { Part-solid GGN at detection } & 12.7 \pm 6.2(4.6-34.3) \\ \quad \text { Number } & \\ \quad \text { Size mm } & \end{array}$

Data are presented as median (interquartile range) or mean \pm SD (range), unless otherwise stated. SSN: sub-solid nodule; GGN: ground-glass nodule. ${ }^{\#}$ : $n=108$.

Nodule size and type

Pure GGNs

69 SSNs were pure ground glass at the time of detection, including 37 nodules $\geqslant 10 \mathrm{~mm}$ and 32 nodules $<10 \mathrm{~mm}$. 20 nodules (in 20 subjects) developed a solid component, resulting in 49 pure GGNs at the last follow-up CT (table 2).

\section{Part-solid nodules}

48 nodules were part-solid at detection. 30 part-solid GGNs were $\geqslant 10 \mathrm{~mm}$ in diameter and 18 part-solid GGNs were $<10 \mathrm{~mm}$. 20 pure GGNs developed a solid component, resulting in 68 part-solid GGNs at the last follow-up CT (table 2).

\section{Clinical course}

\section{Non-resected nodules}

84 nodules in 75 participants were followed according to the study protocol (table 2). At baseline, 51 were pure GGN and 33 were part-solid. Of the pure GGNs, 13 developed a solid component according to the two radiologists. During follow-up, none of the 84 SSNs developed into a clinical relevant malignancy. Nine (12.0\%) subjects died from pulmonary adenocarcinoma presenting as (another) solid nodule $(\mathrm{n}=6)$, oesophageal carcinoma $(\mathrm{n}=1)$, colon carcinoma $(\mathrm{n}=1)$ and lymphoma $(\mathrm{n}=1)$.

\section{Resected nodules}

33 SSNs were resected, of which 11 were pure GGNs and 22 were part-solid GGNs (table 2). Five SSNs turned out to be benign, including our only case of atypical adenomatous hyperplasia. Nine SSNs were preinvasive lesions (AIS, originally diagnosed as bronchoalveolar carcinoma) six were pure GGNs (mean size $15.2 \pm 3.1 \mathrm{~mm}$ ) and three were part-solid GGNs (mean size $16.9 \pm 3.3 \mathrm{~mm}$ ). This difference in size was not significant $(\mathrm{p}=0.21)$ (table 2). In this group of nine preinvasive lesions, three SSNs had only visual progression according to the trial radiologist.

19 SSNs were diagnosed as invasive adenocarcinomas, of which six had only visual progression according to the trial radiologist. Among the 19 invasive carcinomas there were four pure GGNs (mean size $12.9 \pm 1.2 \mathrm{~mm}$ ) and 15 part-solid GGNs (mean size $17.5 \pm 9.2 \mathrm{~mm}$ ). This difference in size was not significant $(\mathrm{p}=0.12)$.

TABLE 2 Management and histology of all subsolid nodules at the last computed tomography

\begin{tabular}{|c|c|c|c|c|c|c|}
\hline & Non-resected & Resected & Benign & AIS & Invasive carcinoma & Total \\
\hline Persistent pure GGN & 38 & 11 & 1 & 6 & 4 & 49 \\
\hline Persistent part-solid GGN & 33 & 15 & 4 & 1 & 10 & 48 \\
\hline Total & 84 & 33 & 5 & 9 & 19 & 117 \\
\hline
\end{tabular}

Data are presented as n. AIS: adenocarcinoma in situ; GGN: ground-glass nodule. 
All but one invasive carcinoma was stage IA $(n=16)$ or IB $(n=2)$. One invasive carcinoma was a stage IV at time of resection, which was delayed because of a competing malignancy. In this group of participants with a resected SSN, four (12.1\%) participants died of lung cancer.

\section{Growth rates}

Eight participants with 11 SSNs were referred and had their SSN resected after the first detection. These participants consequently had no follow-up CT. In five other participants with five nodules, changes in size could not be assessed because of missing data in three or inadequate segmentation of the nodule in two cases, leaving 101 nodules in 98 participants available for growth-rate analysis (table 3). 22 SSNs were stable or decreased in size and 79 nodules showed growth (table S4 in the online supplementary material).

Relative increase in mass of the nodule as compared to the first appearance is graphically displayed in figure 1. Two of the $13 \mathrm{SSN}$ that were not resected, showed a minimal increase in size that, in combination with a short follow-up time, resulted in an MDT $<400$ days. Except for three benign nodules (rapidly increasing infections), all nine fast-growing SSNs (MDT $<400$ days) were invasive or pre-invasive. However, most invasive tumours were slow growing: 16 (84.2\%) out of 19 had an MDT $\geqslant 400$ days, including one case with a growth $<30 \%$ (table 3 ).

\section{Follow-up protocol}

Only nine SSNs showed $\geqslant 30 \%$ increase in mass and an MDT $<400$ days, including three benign cases, three AIS and three invasive carcinomas (table 3). The vast majority of invasive carcinomas had an MDT $>400$ days, making this cut-off inappropriate to discriminate between benign and malignant subsolid nodules. Table 3 shows the number of nodules that showed $\geqslant 30 \%$ increase in size compared with baseline (i.e. mass on the first CT on which the nodule could be detected). Note that only one invasive carcinoma showed $<30 \%$ increase in mass while none of the preinvasive lesions showed $<30 \%$ increase in mass. The invasive carcinoma that showed $<30 \%$ increase in mass, however, showed a significant increase in size of the solid component and was therefore referred and resected.

\section{Discussion}

In the current analysis, we describe our experience with close follow-up of SSNs and further evaluation of growing SSNs and SSNs with a new or growing solid component (figs 2 and 3). This approach was found to be safe in a large lung cancer screening trial. We also report that, when using a cut-off of $30 \%$ increase in mass and/or volume of the solid component, no clinically relevant carcinomas would have been missed during a median follow-up of 95 months.

Recently KIM et al. [22] found an interscan variability of $-17.7 \%-18.6 \%$ for the mass of SSNs with solid portions $\leqslant 5 \mathrm{~mm}$, which we find supportive of our chosen cut-off value of $30 \%$ for significant growth.

\section{TABLE 3 Management and histology of persistent decreasing, stable and growing subsolid} nodules

\begin{tabular}{|c|c|c|c|c|}
\hline & \multirow[t]{2}{*}{ Growth $<30 \%{ }^{\#}$} & \multicolumn{2}{|c|}{ Growth $\geqslant 30 \% \pi$} & \multirow[t]{2}{*}{ Total } \\
\hline & & Slow $^{+}$ & Fast $^{\S}$ & \\
\hline \multicolumn{5}{|l|}{ Non-solid $f$} \\
\hline Non-resected & 13 & 15 & 1 & 29 \\
\hline Benign & 2 & 1 & & 3 \\
\hline AIS & & 4 & 1 & 5 \\
\hline Invasive & & 2 & 2 & 4 \\
\hline Subtotal & 15 & 22 & 4 & 41 \\
\hline \multicolumn{5}{|l|}{ Part-solid $f$} \\
\hline Non-resected & 24 & 16 & & 40 \\
\hline Benign & & & 2 & 2 \\
\hline AIS & & 1 & 2 & 3 \\
\hline Invasive & 1 & 13 & 1 & 15 \\
\hline Subtotal & 25 & 30 & 5 & 60 \\
\hline Total & 40 & 52 & 9 & 101 \\
\hline \multicolumn{5}{|c|}{$\begin{array}{l}\text { AIS: adenocarcinoma in situ. }{ }^{\#} \text { : including } 22 \text { subsolid nodules that were stable or decreased in size; } \\
\text { ๆ: increase in mass compared with baseline computed tomography (i.e. the first computed tomography on } \\
\text { which the nodule could be detected); }{ }^{+} \text {: mass doubling time } \geqslant 400 \text { days; }{ }^{\S} \text { : mass doubling time <400 days; } \\
f: \text { nature of the subsolid nodule as assessed on the last computed tomography scan. }\end{array}$} \\
\hline
\end{tabular}



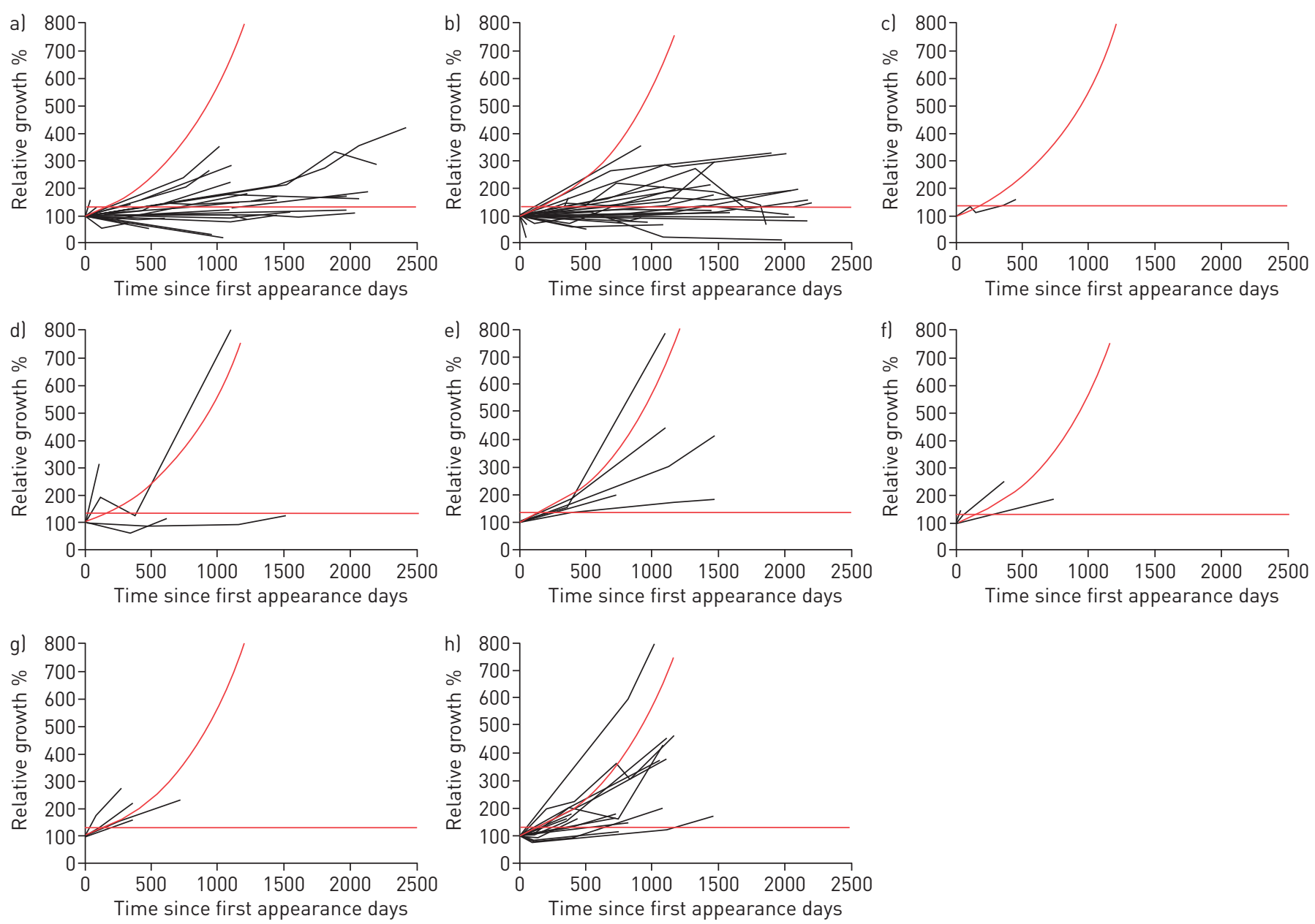

FIGURE 1 Growth patterns on the persistent subsolid nodules in the trial. a) non-resected pure ground-glass nodule; b) non-resected part-solid ground-glass nodule; c) resected benign pure ground-glass nodule; d) resected benign part-solid ground-glass nodule; e) resected adenocarcinoma in situ pure ground-glass nodule; f) resected adenocarcinoma in situ part-solid ground-glass nodule; g) resected invasive pure ground-glass nodule; h) resected invasive part-solid ground-glass nodule. On the $\mathrm{X}$-axis, the number of days since the first appearance; on the $\mathrm{Y}$-axis, the relative growth as compared with the first appearance. The horizontal line represents a relative increase of mass of $30 \%$. The curved line represents a mass doubling time of 400 days.
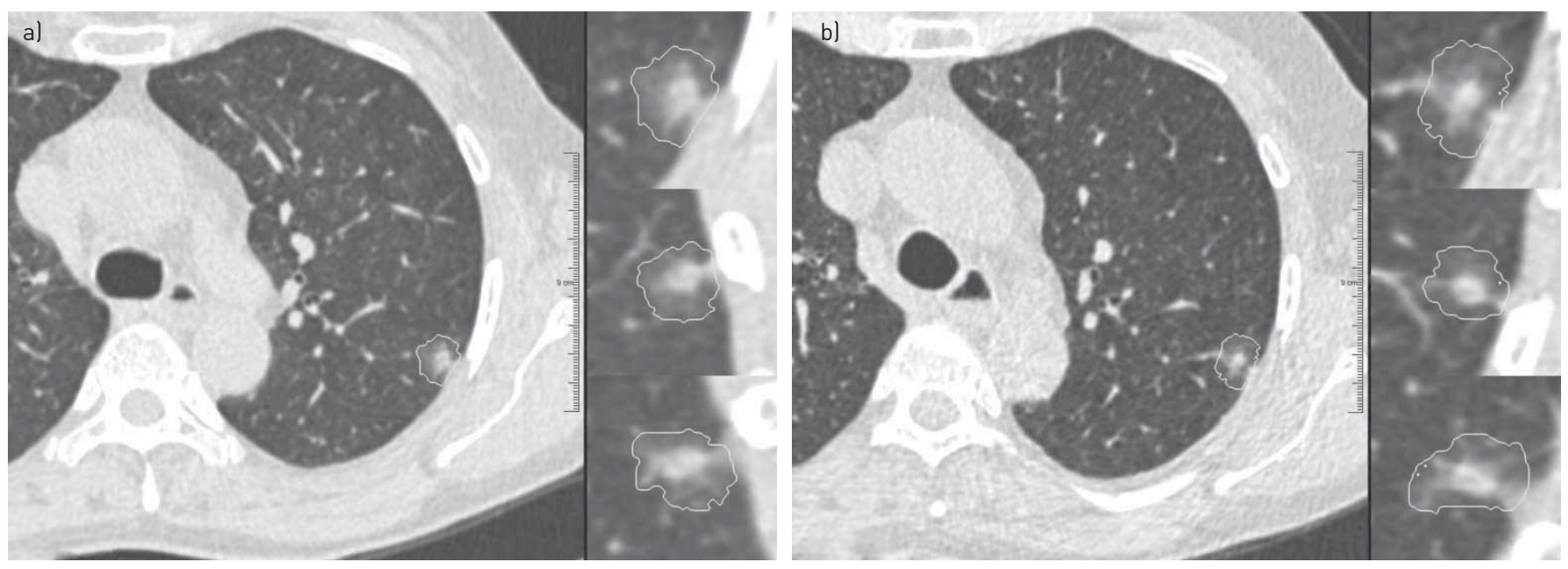

FIGURE 2 Illustration of a stable part-solid ground-glass nodule. a) January 2006; b) September 2008. The female trial participant was 57 years of age in 2006 and she was followed up until November 2012. The images show an axial cross-section with magnified axial (top), coronal (middle) and sagittal (bottom) reconstructions. According to the Fleischner recommendations, this nodule would have been resected. 

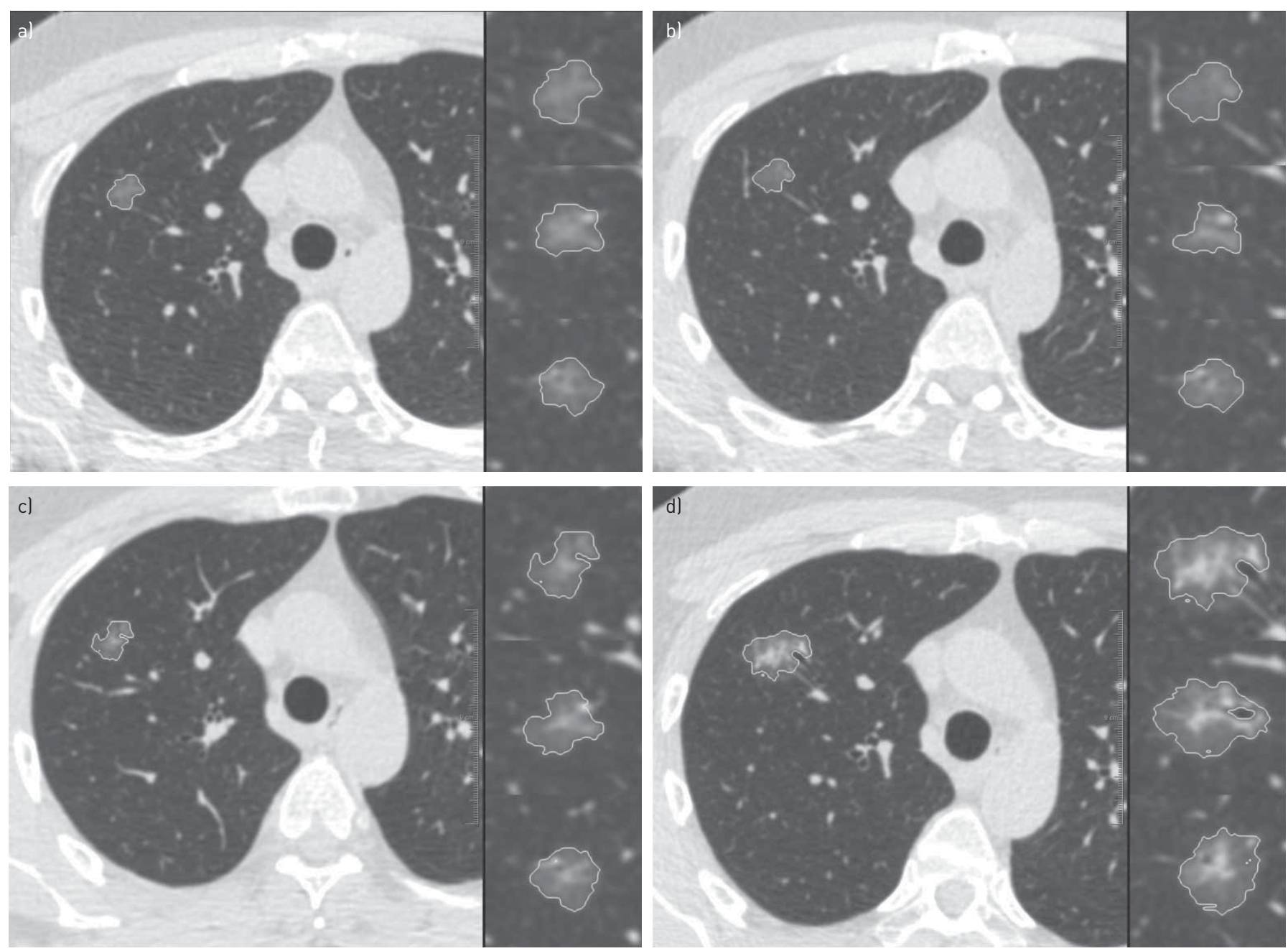

FIGURE 3 Illustration of a pure ground-glass nodule which developed a solid component. a) June 2004; b) October 2004; c) June 2005; d) July 2007. The male trial participant was 57 years of age in 2004 and he was followed up until November 2012. The images show an axial cross-section with magnified axial (top), coronal (middle) and sagittal (bottom) reconstructions. The nodule was classified as a pure ground-glass nodule from the first three computed tomography images. The histological diagnosis was adenocarcinoma in situ.

For persistent part-solid GGNs with a solid component $>5 \mathrm{~mm}$, the new recommendations from the Fleischner Society are fairly aggressive because of their high likelihood of malignancy [1]. We showed, however, that close follow-up may be a safe approach even though it is likely that many of the part-solid GGNs that were followed would have been diagnosed as malignant when resected. None of part-solid GGNs that were not resected developed into clinically relevant lung cancer in our cohort.

For pure GGNs $>5 \mathrm{~mm}$, the Fleischner Society recommends further evaluation in case of nodule growth. In the NELSON trial, the decision to refer a SSN to the pulmonologist for further evaluation was based on a $25 \%$ increase in volume calculated from the diameter as measured with electronic callipers, irrespective of the baseline character of the nodule. However, in 15 cases, there was visual progression only as assessed by the trial screening radiologist either in size or density of the SSN or size of the solid component. In nine of these cases, the nodule turned out to be either AIS $(n=3)$ or invasive adenocarcinoma $(n=6)$. We therefore retrospectively measured volume and mass of all SSNs and reported that a total increase in mass $<30 \%$ seems to be a reliable parameter to exclude clinical relevant malignancies, however for an increase $\geqslant 30 \%$ there is great overlap between malignant and non-malignant SSNs.

For cancer screening to be effective, a good balance between the benefits and harms is required. One of the potential harms is the extent of overdiagnosis. This issue is still topic of debate in breast cancer screening $[23,24]$. Overdiagnosis has been reported to be a problem in lung cancer screening as well [2527]. In lung cancer screening, detection and treatment of cancers with a VDT $\geqslant 400$ days is often considered overdiagnosis [27]. When using this definition, detection and treatment of the vast majority of SSNs can be considered overdiagnosis despite their high likelihood of containing malignant cells. 
In the current investigation, we retrospectively used MDT instead of VDT. Mass is a parameter that integrates volume and density, and will therefore not only increase when volume increases, but also when a solid component within a lesion develops or progresses. De Hoop et al. [28] showed that mass is a more sensitive parameter to detect progression than volume in SSNs and showed that even in the group of invasive carcinomas, the vast majority had an MDT $>400$ days [28].

In one case of invasive carcinoma, mass did not increase, even though the solid component increased in size, because total size of the nodule decreased. Therefore, volumetry of the solid component is proposed as an additional indicator of growth of the nodule.

Recently LeE et al. [29] described their use of CT features to differentiate between invasive pulmonary adenocarcinomas and preinvasive lesions in SSNs. They observed that in pure GGNs, a cut-off value of $10 \mathrm{~mm}$ was optimal for differentiation between a preinvasive lesions and invasive adenocarcinomas with a sensitivity of $55.3 \%$ and a specificity of $100 \%$. Lim et al. [30] described a cut-off value of $16.4 \mathrm{~mm}$ in a series of 46 resected pure GGNs for differentiating invasive adenocarcinomas from preinvasive lesions. We cannot confirm the findings of LEE et al. [29] since none of our resected nodules measured less than $10 \mathrm{~mm}$ and, in contrast with LeE et al. [29] and Lim et al. [30], we did not find a significant difference in size between preinvasive and invasive lesions. In the group of part-solid nodules, invasive adenocarcinomas were significantly larger the preinvasive lesions in the series of LeE et al. [29]. We also found a larger size of invasive part-sold lesion compared to preinvasive lesions; however, this difference was not significant in our series, which can be attributed to our small number of only three part-solid preinvasive lesions.

PET-CT was not a routine part of the diagnostic work-up of referred SSNs in our series. The Fleischner Society states that PET is of limited value and even potentially misleading for pure GGNs, and they only recommend considering PET-CT for part-solid nodules $>10 \mathrm{~mm}$ [3]. PET-CT has been shown to be a significant predictor of surgical outcomes and to be important to determine the appropriateness of sublobar resection in cases of stage $1 \mathrm{~A}$ adenocarcinoma of the lung [31].

For the management of SSNs one would prefer to be able to predict the lifelong behaviour for SSNs, but that is a challenge that requires larger sample size and longer follow-up. Until this challenge has been addressed, the disadvantages of repeated follow-up must be weighed up with the disadvantages of invasive treatment. Invasive treatment must take into account that enough lung tissue is preserved as subjects often have pulmonary comorbidity and multiple lesions can be present or develop. As limited surgery, stereotactic radiotherapy and percutaneous interventions allow treatment of multiple lesions and preservation of lung function, such treatment remains a consideration. In their recent paper on radio frequency ablation for ground glass opacity dominant lung adenocarcinoma, KodAma et al. [32] conclude that this is a feasible, safe and useful therapeutic option to control these carcinomas.

For many participants, low-frequency follow-up with low-dose CT may be preferable for the near future, because these subjects may well be at high risk of developing new solid nodules and SSNs.

The major strengths of the current analysis were the relative large size of the study population of this relatively rare, but important, entity and the relative long follow-up.

The study also suffers from limitations. First, we do not know the exact malignancy rate among the studied SSNs, since the majority of the SSNs were not resected. Secondly, even from those participants, we had stage IA or stage IB at time of resection, three subjects died during follow-up. Thirdly, our results including the cut-off value of $30 \%$ mass increase need external validation, preferably in a prospective study.

In conclusion, persistent SSNs have a high malignancy rate according to pathological analysis but rarely develop into clinical manifest malignancies unexpectedly. Our data suggest that long-term follow-up with CT may be a safe option to monitor changes in persistent SSNs. We like to suggest that (minimally) invasive intervention could be considered only in SSNs that show $\geqslant 30 \%$ growth or a new appearing or growing solid component.

\section{References}

1 Henschke CI, Yankelevitz DF, Mirtcheva R, et al. CT screening for lung cancer: frequency and significance of part-solid and nonsolid nodules. AJR Am J Roentgenol 2002; 178: 1053-1057.

2 Sone S, Nakayama T, Honda T, et al. Long-term follow-up study of a population-based 1996-1998 mass screening programme for lung cancer using mobile low-dose spiral computed tomography. Lung Cancer 2007; 58: 329-341.

Naidich DP, Bankier AA, MacMahon $\mathrm{H}$, et al. Recommendations for the management of subsolid pulmonary nodules detected at CT: a statement from the Fleischner Society. Radiology 2013; 266: 304-317.

4 Oh JY, Kwon SY, Yoon HI, et al. Clinical significance of a solitary ground-glass opacity (GGO) lesion of the lung detected by chest CT. Lung Cancer 2007; 55: 67-73.

5 Park CM, Goo JM, Lee HJ, et al. Nodular ground-glass opacity at thin-section CT: histologic correlation and evaluation of change at follow-up. Radiographics 2007; 27: 391-408. 
6 Aoki T, Nakata H, Watanabe H, et al. Evolution of peripheral lung adenocarcinomas. Am J Roentgenol 2000; 174 763-768.

7 Hasegawa M, Sone S, Takashima S, et al. Growth rate of small lung cancers detected on mass CT screening. $\mathrm{Br} \mathrm{J}$ Radiol 2000; 73: 1252-1259.

8 Noguchi M, Morikawa A, Kawasaki M, et al. Small adenocarcinoma of the lung. Histologic characteristics and prognosis. Cancer 1995; 75: 2844-2852.

9 Nakajima R, Yokose T, Kakinuma R, et al. Localized pure ground-glass opacity on high-resolution CT: histologic characteristics. J Comput Assist Tomogr 2002; 26: 323-329.

10 Nakata M, Sawada S, Saeki H, et al. Prospective study of thoracoscopic limited resection for ground-glass opacity selected by computed tomography. Ann Thorac Surg 2003; 75: 1601-1605.

11 Seki N, Sawada S, Nakata M, et al. Lung cancer with localized ground-glass attenuation represents early-stage adenocarcinoma in nonsmokers. J Thorac Oncol 2008; 3: 483-490.

12 Suzuki K, Asamura $\mathrm{H}$, Kusumoto $\mathrm{M}$, et al. "Early" peripheral lung cancer: prognostic significance of ground glass opacity on thin-section computed tomographic scan. Ann Thorac Surg 2002; 74: 1635-1639.

13 Ost D, Fein AM, Feinsilver SH. Clinical practice. The solitary pulmonary nodule. $N$ Engl J Med 2003; 348: 2535-2542.

14 Usuda K, Saito Y, Sagawa M, et al. Tumor doubling time and prognostic assessment of patients with primary lung cancer. Cancer 1994; 74: 2239-2244.

15 Winer-Muram HT, Jennings SG, Tarver RD, et al. Volumetric growth rate of stage I lung cancer prior to treatment: serial CT scanning. Radiology 2002; 223: 798-805.

16 Yankelevitz DF, Henschke CI. Does 2-year stability imply that pulmonary nodules are benign?. Am J Roentgenol 1997; 168: 325-328.

17 Yankelevitz DF, Reeves AP, Kostis WJ, et al. Small pulmonary nodules: volumetrically determined growth rates based on CT evaluation. Radiology 2000; 217: 251-256.

18 van Klaveren RJ, Oudkerk M, Prokop M, et al. Management of lung nodules detected by volume CT scanning. $N$ Engl J Med 2009; 361: 2221-2229.

19 van Iersel CA, de Koning HJ, Draisma G, et al. Risk-based selection from the general population in a screening trial: selection criteria, recruitment and power for the Dutch-Belgian randomised lung cancer multi-slice CT screening trial (NELSON). Int J Cancer 2007; 120: 868-874.

20 Kuhnigk JM, Dicken V, Bornemann L, et al. Morphological segmentation and partial volume analysis for volumetry of solid pulmonary lesions in thoracic CT scans IEEE Trans. Med Imaging 2006; 25: 417-434.

21 Scholten ET, Jacobs C, van Ginneken B, et al. Computer aided segmentation and volumetry of artificial ground glass nodules on chest computed tomography. Am J Roentgenol 2013; 201: 295-300.

22 Kim H, Park CM, Woo S, et al. Pure and part-solid pulmonary ground-glass nodules: measurement variability of volume and mass in nodules with a solid portion less than or equal to $5 \mathrm{~mm}$. Radiology 2013; 269: 585-593.

23 Independent UK. Panel on Breast Cancer Screening. The benefits and harms of breast cancer screening: an independent review. Lancet 2012; 380: 1778-1786.

24 Wise J. Mammography results in substantial overdiagnosis of breast cancer, concludes study. BMJ 2012; 345: e7910.

25 Reich JM. A critical appraisal of overdiagnosis: estimates of its magnitude and implications for lung cancer screening. Thorax 2008; 63: 377-383.

26 Bach PB. Overdiagnosis in lung cancer: different perspectives, definitions, implications. Thorax 2008; 63: 298-300.

27 Yankelevitz DF, Kostis WJ, Henschke CI, et al. Overdiagnosis in chest radiographic screening for lung carcinoma: frequency. Cancer 2003; 97: 1271-1275.

28 De Hoop B, Gietema H, van de Vorst S, et al. Pulmonary ground-glass nodules: increase in mass as an early indicator of growth. Radiology 2010; 255: 199-206.

29 Lee SM, Park CM, Goo JM, et al. Adenocarcinomas versus preinvasive lesions appearing as ground-glass nodules: differentiation by using CT features. Radiology 2013; 268: 265-273.

30 Lim HJ, Ahn S, Lee KS, et al. Persistent pure ground-glass opacity lung nodules $\geqslant 10 \mathrm{~mm}$ in diameter at CT scan: histopathologic comparisons and prognostic implications. Chest 2013; 144: 1291-1299.

31 Okada M, Nakayama H, Okumura S, et al. Multicenter analysis of high-resolution computed tomography and positron emission tomography/computed tomography findings to choose therapeutic strategies for clinical stage IA lung adenocarcinoma. J Thorac Cardiovasc Surg 2011; 141: 1384-1391.

32 Kodama H, Yamakado K, Hasegawa T, et al. Radiofrequency ablation for ground-glass opacity-dominant lung adenocarcinoma. J Vasc Interv Radiol 2014; 25: 333-339. 